\title{
Efavirenz' adverse reactions: a possible link between depression and gynecomastia
}

\author{
Ioan Alexandru Diaconu ${ }^{1 *}$, Daniela Adriana Ion², Victoria Aramă ${ }^{1,2}$, Adriana Hristea ${ }^{1,2}$, Ruxandra Moroti ${ }^{1,2}$ \\ From The 10th Edition of the Scientific Days of the National Institute for Infectious Diseases "Prof Dr Matei \\ Bals" \\ Bucharest, Romania. 15-17 October 2014
}

\section{Background}

Reports show that $1.8-8.4 \%$ of male patients receiving efavirenz (EFV) develop gynecomastia by unclear multiple mechanisms, one of them being a direct binding to estrogen receptors. However, EFV can independently cause gynecomastia by increasing prolactin levels and we postulate that this mechanism is a dopaminemediated one. Dopamine is a strong prolactin inhibitor. Conversely, a lack of dopamine can cause hyperprolactinemia. On the other hand, dopamine is an antidepressant neurotransmitter and depression is one of the very frequent adverse reactions to EFV. We present the case of an HIV-positive newly diagnosed male patient who developed mild depression and amplified a preexistent gynecomastia after the introduction of an EFV-containing antiretroviral regimen.

\section{Case report}

A 41-year-old male patient, diagnosed with CDC-A2 HIV infection, had been receiving antiretroviral treatment (ART) for 14 months with $\mathrm{ABC}+3 \mathrm{TC}+\mathrm{EFV}$ and had been favorably evaluated regarding the immuno-virological course. Nevertheless, after 6 months of ART he developed bilateral gynecomastia with mastodynia and he reported also mild depression. A careful anamnesis discovered a previous mild gynecomastia with also mild rising in prolactin level. Current clinical examination showed moderate bilateral concentric gynecomastia. The breast echography was normal. Brain MRI was performed and can't exclude microprolactinoma. Lab findings showed normal levels of estrogen and testosterone and high levels of prolactin. We considered dropping
EVF and replacing it with raltegravir. After switching (raltegravir doesn't affect the endocrine ax) mastodynia ceased in one month, the breast volume regressed in 2-3 months and the prolactin level decreased. Besides, the depression's symptoms diminished.

\section{Conclusion}

In the presented case, gynecomastia was related to hyperprolactinemia and the last was related to EFV use. Moreover, the patient presented also an EFV related depression. It makes sense to consider a unique mechanism for both EFV's adverse reactions: a drop in dopamine-level caused by EFV, which produce depression and also a hyperprolactinemia with gynecomastia.

\section{Acknowledgement \\ This paper is realized as a part of doctoral projects - POSDRU/159/1.5/S/ 137390.}

\section{Authors' details}

'National Institute for Infectious Diseases "Prof. Dr. Matei Balş", Bucharest, Romania. ${ }^{2}$ Carol Davila University of Medicine and Pharmacy, Bucharest, Romania.

\section{Published: 15 October 2014}

\section{doi:10.1186/1471-2334-14-S7-P27}

Cite this article as: Diaconu et al:: Efavirenz' adverse reactions: a possible link between depression and gynecomastia. BMC Infectious Diseases 2014 14(Suppl 7):P27.

\footnotetext{
* Correspondence: diaconuia@yahoo.com

'National Institute for Infectious Diseases "Prof. Dr. Matei Balş", Bucharest, Romania

Full list of author information is available at the end of the article
} 